\title{
Management of thigh lipofibromatosis in a newborn: a case report
}

YL Lam ${ }^{1 *}$, MB, ChB, FHKAM (Orthopaedic Surgery), WY Ho ${ }^{1}$, MB, BS, FHKAM (Orthopaedic Surgery), Raymond Yau', MB, BS, FHKAM (Orthopaedic Surgery), Victor WK Lee ${ }^{2}$, MB, BS, Tony WH Shek², MB, BS, FHKAM (Pathology)

\author{
${ }^{1}$ Department of Orthopaedics and Traumatology \\ ${ }^{2}$ Department of Pathology \\ Queen Mary Hospital, Pokfulam, Hong Kong
}

*Corresponding author: albertlam2000@yahoo.com

Hong Kong Med J 2019;25:68-70

https://doi.org/10.12809/hkmj166060

\section{Case report}

A $3.6 \mathrm{~kg}$ (gravida 2 para 2 full-term normal vaginal delivery) baby boy presented to Queen Mary Hospital, Hong Kong in September 2015 with a painless swelling on the left thigh on day 2 after birth. The antenatal check-up had been normal. Physical examination revealed a $2-\times 2-\mathrm{cm}$ intramuscular swelling over the lateral aspect of the left thigh. There was mild tenderness but no increase in local temperature, skin change, or thrill. Limb movements were normal and symmetrical. Distal pulse and tissue circulation were also normal. Further systemic examination revealed no syndromal features, other swelling, or other significant findings on other systems.

Blood tests revealed slightly deranged white cell count and haemoglobin, creatine kinase, lactate dehydrogenase, and C-reactive protein levels. Renal and liver function tests and calcium and phosphate levels were normal. Radiographs did not show any abnormality. Ultrasonographic study on day 2 after birth revealed a swollen and oedematous distal part of the left vastus lateralis muscle. The muscular architecture was preserved. There was no definite mass and the vascular signal was also normal. A repeat ultrasonographic study in week 2 after birth showed an ill-defined intramuscular echogenic lesion at the distal left vastus lateralis muscle. A magnetic resonance imaging scan at age 2 months showed an infiltrative lesion of $3 \times 4 \times 4.3 \mathrm{~cm}$ in the vastus lateralis muscle with extension to the short head of the biceps muscle. It was heterogeneously hyperintense to the muscle on both T1- and T2weighted images.

An image-guided wide-bore needle biopsy revealed a benign-looking lesion composed of fascicles of spindle cells admixed with lobules of mature fat. The differential diagnoses included lipofibromatosis, fibrous hamartoma of infancy, or less likely, calcifying aponeurotic fibroma, and lipoblastoma or lipoblastomatosis.

During the course of the investigation, there was deterioration of knee range in extension. At age 5 months, the flexion contracture was 25 degrees. Clinically, there was size progression. In addition, the parents were concerned about the subjective increase in tumour size. After discussion of the treatment options with the parents, a marginal excision was performed at age 5 months.

Microscopic examination of the surgical specimen showed thick fascicles of bland-looking spindle cells traversing a large number of mature adipocytic lobules, concentrated around the septal region (Figs 1 and 2). Infiltration around the skeletal muscle was noted. There was no organoid nest of mesenchymal cells to suggest fibrous hamartoma of infancy, nor any calcification foci to suggest calcifying aponeurotic fibroma. Mitosis was inconspicuous. Necrosis was absent. Immunohistochemical staining of the spindle cells showed CD34+, S100+, and actin patchy+, whereas beta-catenin, epithelial membrane antigen, desmin, and c-kit were negative. A diagnosis of lipofibromatosis was made.

The patient was given postoperative physiotherapy as maintenance for his knee range of motion. The latest follow-up was at 5 months

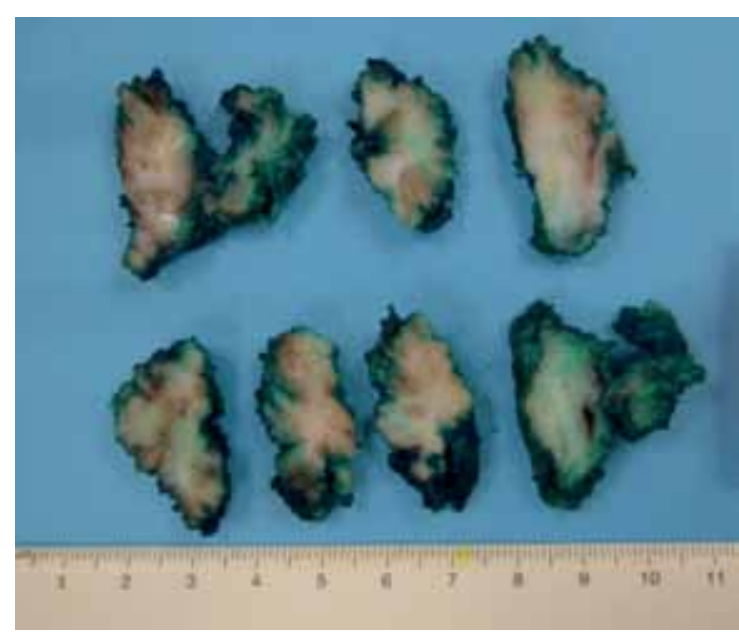

FIG I. Gross specimen (serially sectioned, periphery inked green) showing admixture of whitish area, tan skeletal muscle, and yellowish fat 


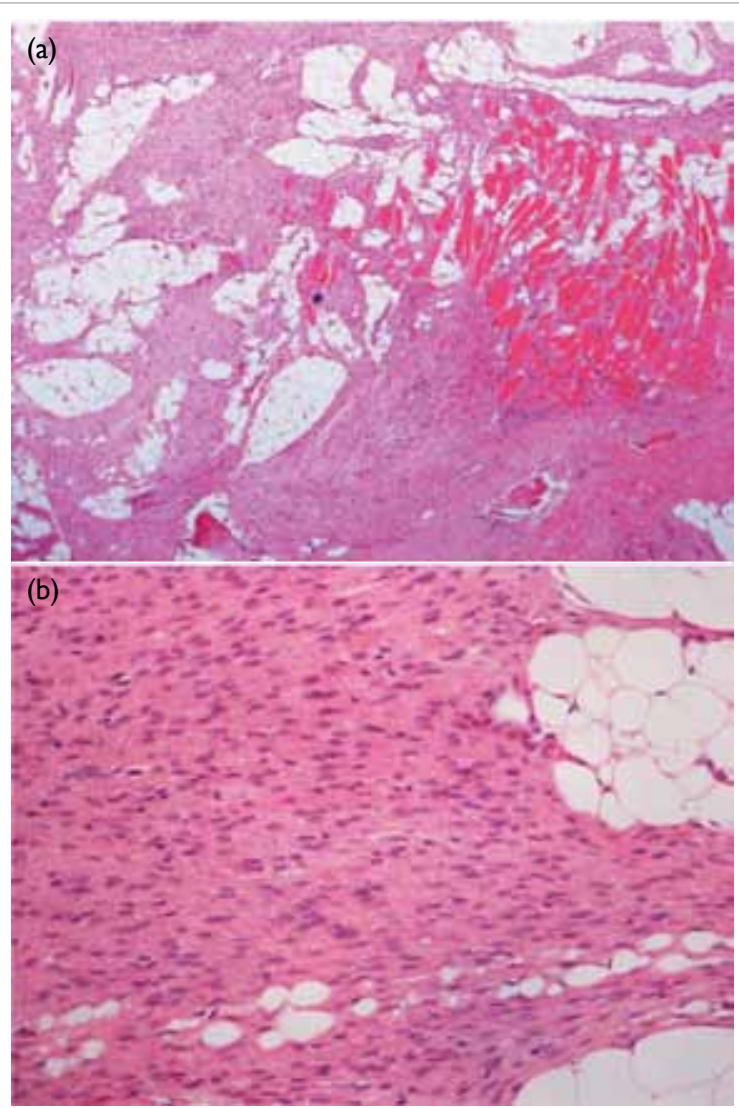

FIG 2. (a) Thick fascicles of spindle cells traversing mature fat lobules, with infiltration of skeletal muscle (right half); (b) bland-looking cytology of the spindle cells in fascicles on higher-power magnification postoperatively (age 10 months). He was able to pull himself to a standing position. He could walk with support for more than 10 steps. Thickened scarring could be felt under the old incision wound. His latest knee range was 0 to 130 degrees (the same as the right side).

\section{Discussion}

The reported occurrence of neonatal tumours is one in 12500 to 27500 live births although there is little information on the real incidence. It has not been well studied because of its rarity. ${ }^{1}$ Soft tissue tumours account for $25 \%$ of all neoplasms in infancy, of which approximately $15 \%$ are malignant. ${ }^{2}$

It is quite impossible to clinically differentiate between a benign and a malignant tumour in a child. Further investigation by means of ultrasonography or magnetic resonance imaging may provide a definitive diagnosis in certain conditions such as haemangioma but may be impossible without histological studies. ${ }^{1}$

More confusing, the behaviour of the tumour in this specific patient group may not be the same as in their adult counterparts. The natural history of some disease entities has not been documented. Even a benign lesion may invade locally, causing symptoms such as developmental deformity and even death. ${ }^{1}$

Watchful neglect may be a treatment option for some lesions with a potential of spontaneous regression such as haemangioma. ${ }^{2}$ In the other conditions, surgical resection may be an option for management of a progressing disease.

There are four possible diagnoses based on the first wide-bore needle biopsy finding. Calcifying aponeurotic fibroma ${ }^{3}$ is tumour characterised by foci of calcification, palisaded round cells and radiating arms of fibroblasts. This lesion has a high tendency of local recurrence (50\%). The natural history is tumour growth reduction with age. Conservative resection or palliative resection should be considered.

Lipoblastoma or the diffuse subtype lipoblastomatosis ${ }^{3}$ is a benign tumour and resembles fetal fat tissue. The solitary lipoblastoma is usually in the subcutaneous area and lipoblastomatosis may invade deep structures. Local recurrence may occur in $9 \%$ to $22 \%$ of patients especially in the diffuse subtype.

Fibrous hamartoma of infancy ${ }^{3}$ is a benign infiltrative lesion. It is a rapidly growing mass in the subcutaneous or dermis of the axillary fold, shoulder, arm, forearm, back, groin or thigh. It is characterised by well-defined fibrocollagenous tissue, small rounded primitive mesenchymal cells, and mature fat. Local excision is the treatment of choice. Recurrence is rare.

Lipofibromatosis, ${ }^{3}$ also known as nondesmoid-type infantile fibromatosis, is a very rare paediatric disease. To date, approximately only 60 cases have been reported. Typically, it is an ill-defined slow-growing tumour that presents as a painless lesion in the limbs. The rate of local recurrence is high. The regrowth and persistent disease rate is $72 \%{ }^{4}$ Fortunately, there is no known metastasis.

These four diagnoses differ quite significantly in prognosis and recommended management. In the present case, we advised conservative resection for histology, prognosis, management of disease progression, and treatment of the knee flexion contracture.

The diagnosis of a tumour in the neonatal period causes significant psychological distress to parents. $^{5}$ Understanding the natural history and prognosis is the first step towards alleviating parental anxiety followed by inviting them to participate in the planning of future management. Sincere communication is an essential part of patient management. Sometimes professional intervention in the form of psychological support should also be considered. 


\section{Author contributions}

All authors had full access to the data, contributed to the study, approved the final version for publication, and take responsibility for its accuracy and integrity.

Concept or design: YL Lam, WY Ho, R Yau, TWH Shek. Acquisition of data: YL Lam, VWK Lee, TWH Shek.

Analysis or interpretation of data: YL Lam, VWK Lee, TWH Shek.

Drafting of the manuscript: YL Lam, TWH Shek.

Critical revision for important intellectual content: All authors.

\section{Acknowledgement}

We thank Ms Carol Anne Higgins for proofreading the manuscript.

\section{Conflicts of interest}

All authors have disclosed no conflicts of interest.

\section{Funding/support}

This research received no specific grant from any funding agency in the public, commercial, or not-for-profit sectors.

\section{Ethics approval}

This study was approved by the institutional review board (Ref. UW15/414).

\section{References}

1. Moore SW, Satgé D, Sasco AJ, Zimmermann A, Plaschkes J. The epidemiology of neonatal tumours, report of international working group. Pediatr Surg Int 2003;19:50919

2. Palumbo JS, Zwerdling T. Soft tissue sarcomas of infancy. Semin Perinatol 1999;23:299-309.

3. Fletcher CD, Bridge JA, Hogendoorn PC, Mertens F. WHO Classification of Tumours. Lyon: IARC; 2013.

4. Fetsch JF, Miettinen M, Laskin WB, Michal M, Enzinger FM. A clinicopathological study of 45 pediatric soft tissue tumors with an admixture of adipose tissue and fibroblastic elements, and a proposal for classification as lipofibromatosis. Am J Surg Pathol 2000;24:1491-500.

5. Orbach D, Sarnacki S, Brisse HJ, et al. Neonatal cancer. Lancet Oncol 2013;14:e609-20. 